\title{
The Free Software Alternative:
}

\section{Freeware, Open-source software,}

and Libraries

\section{ABSTRACT}

This paper will introduce the reader to the world of freeware and open-source software. Following a brief introduction, the author presents an overview of these types of software. Next comes a discussion of licensing issues unique to freeware and open-source software, which leads directly to issues of registration. The author then offers several strategies readers can adopt to locate these software packages on the Web. The author then addresses questions regarding the use of freeware and open-source software before offering a few closing thoughts.

\section{INTRODUCTION}

I first recognized the potential savings in time, money, and labor offered to librarians and others by freeware and open-source software while I was Head of Technical and Automated Services at St. Ambrose University in Davenport, Iowa. Among other responsibilities, I oversaw the cataloging and processing of all new library materials. Normally, I created original records on OCLC whenever I cataloged. One of the tools I needed to complete this work (particularly with foreign language materials) was an ASCII chart to provide instructions for making characters not found in English, such as $\beta, i$, and ç. Today a search for such symbols is relatively easy (most can be found on any character map), but twenty years ago, it presented more of a challenge. I spent many fruitless hours looking for the implement I needed.

After much searching, I discovered the right tool-David Lord's Ascii Chart. This freeware program featured several tables - one for ANSI characters, control characters, an EBDIC table (Extended Binary Coded Decimal Interchange Code), Palette (for colors), and a list of IBM PC characters. The first and last charts were the ones I referred to the most. Whenever I needed a special symbol in a Windows-based program, the IBM PC chart gave me the formula I needed to make it. Any time I was in DOS or a DOS-based utility (as I was with OCLC), I consulted the ANSI chart to form a diacritical mark. Ascii Chart was a great piece of software that saved me many hours of work and helped me formulate accurate documents and records.

In another instance, shortly after I assumed my duties as the Director of Library Services at Kansas Wesleyan University, the time clock that registered the work hours of student workers broke down. It was an ancient machine that demanded frequent repairs. And although it dutifully

James E. Corbly (james.corbly@gmail.com), from Austin, Minnesota, has been studying freeware and open-source software for over a decade. 
printed check-in and check-out hours on time cards, it did not calculate the number of hours worked, nor could it prevent such abuses as students punching in and out for one another.

Rather than taking the machine to a repair shop, I sought an alternative method of record keeping. I found one - a computer program from Guia International called Picture TimeClock ${ }^{1}$ which not only registers each student's work hours but also totals them so that monthly summaries of each student's work record can be compiled with increased speed, ease, and accuracy. The software lists among its features a photo identification module that prohibits one student from signing in or out for another. Picture TimeClock is freeware, so no monies were involved in its procurement.

I then took this process a step farther. Each month, I digitized the work record sheets required by the business office so I could employ another freeware program, a PDF editor, to transfer the records from Picture TimeClock onto the digitized time sheets. Together, these two programs reduced the amount of time needed to document student worker hours by $88 \%$ while enhancing the correctness of the submitted records. As an added bonus, I obtained a permanent digital copy of each student's work record which could be readily accessed whenever necessary.

Today, I still rely on freeware and open-source software to solve many of my workaday problems. I utilize these packages for a variety of purposes including Web content mining, manipulating PDF documents, and keeping my computers functioning at their optimal level.

\section{What are Freeware and Open-Source software?}

Among the major types of software (commercial, shareware, adware, etc.), freeware and opensource software are unique. Freeware is copyrighted software given away by its owner (normally the author) for others to use. The author retains sole possession of the copyright, so users cannot alter the software. Freeware authors allow individuals to use their productions in any legal manner but they do not allow anyone to sell the software for a profit. Additionally, many freeware suppliers impose boundaries on the use of their products, restricting their application in commercial endeavors, for example.

Open-source software carries the concept of freeware to its ultimate conclusion. With open source products, the copyright holder gives others the right to study, modify, and distribute the software free of charge to anyone for any purpose. Quite often, open source products result from the collaborative efforts of contributors living in numerous locations around the world. Raw program code, along with the compiled program, is available to anyone who is willing to obtain it, scrutinize it, and make additions or improvements to it in the expectation that the combined efforts of many people will result in a product increasingly useful and reliable to end users. Although some open source products lack documentation, many (if not most) have active user groups or communities which serve as sources of assistance to users.

To be considered open source, a software product must meet several criteria, among which are the following: 
- The software must be freely available without cost, royalties, or fees of any kind.

- The program must be distributed as source code (for programmers) and compiled code (for end users).

- End users and programmers may alter the program's code.

- The modified source code must be redistributed under the same conditions as the license for the original version of the software. ${ }^{2}$

One must guard against the temptation to equate freeware and open-source software with publicdomain software. This latter category of software is not copyrighted; hence, it is free of all costs and can be employed without any restrictions whatsoever. ${ }^{3}$ Freeware and open-source software possess copyrights.

Although many users may be unfamiliar with the concepts of freeware and open-source software, they nonetheless rely on them every day in their routine tasks. If one uses the Web, one needs to use a Web browser. Those who employ Microsoft's Internet Explorer are using one of the most popular freeware products available, ${ }^{4}$ while Firefox users rely on an open source package. ${ }^{5}$ Many freeware and open source systems are standards in their fields. CCleaner (freeware) has won numerous awards for its efficiency not only as a cleaner and system optimization tool but also as a guardian of user privacy. ${ }^{6}$ Audacity (open source) is a sound recorder and editor employed not only by amateurs but also by professionals in the field. ${ }^{7}$ Access to the Web would be impossible without the use of Apache (open source), the number one HTTP server. ${ }^{8}$

It is also important to note that software can change type. Freeware can become shareware, commercial software can morph into freeware, etc.

\section{LICENSING}

Licensing of open source products is rather straightforward. Although there are over sixty-five different open source licenses, one predominates - the General Public License (GPL). This is the most popular license used for open-source software. The GPL serves as the license for approximately $70 \%$ of open source products.

The GPL first appeared in 1989. Richard Stallman, formerly of the Massachusetts Institute of Technology and founder of the nonprofit Free Software Foundation, authored it. Modifications made to the GPL helped keep it vibrant as the years passed and the information technology world changed. As of this writing, the GPL in use is the third version, which came out on June 29, 2007.

The foundation of the GPL consists of four principles:

1. The right of individuals to use software for any purpose;

2. The right of individuals to alter software to meet individual needs;

3. The right of individuals to share software with others; and 
4. The right of individuals to freely distribute the changes one makes to software. ${ }^{9}$

To these ends, the GPL gives end users the right to freely reproduce and distribute copies of a software program's source code, providing that each copy displays a copyright notice, a disclaimer of warranty, a copy of the GPL, and GPL notices.

The right to modify the software's code and freely distribute it to others, taking care to list all modifications made to the code and insuring that every condition outlined above is met.

Commentators often refer to GPL as "copyleft" licensing. Copyleft is a method of making software freely available and requires all modified versions to meet the criteria already listed. One can read the text of this license at: https://www.gnu.org/licenses/gpl-3.0.txt. ${ }^{10}$

In addition to the GPL, open-source software authors distribute their work under other arrangements such as the Berkeley Software Distribution Licenses (BSDL), the Mozilla Public License, the NASA Open Source Agreement, and the Common Public License.

Freeware licensing is not nearly as uniform as open source. There is no freeware equivalent to the GPL and the rights and responsibilities of the copyright owner vary from program to program.

Having noted this, there are clauses that many freeware licenses hold in common. Among these are the following:

- The copyright to the software is retained either by the author of the software or its provider.

- End users may install the software and use it for any legal purpose.

- One may install and use the software only on a specified number of computers.

- Users may copy and distribute the software provided the original copyright remains intact.

- One cannot charge a fee for copies of the freeware save for distribution costs.

- The software is provided "as is" - the copyright owner assumes no liability for any damage caused by product usage.

- Freeware frequently has usage limits. Many freeware licenses permit only personal or noncommercial use of the product. There may be limits on use of the freeware with other software packages and restrictions on freeware use over a network.

With such a variety of clauses such as these, it is hardly surprising that freeware licenses vary in length. One freeware program I regularly use has a license consisting of three small paragraphs, while another boasts a license five pages in length.

Due to these characteristics of freeware licensing, I always copy the text of a freeware license to a blank page of my word processor. I keep this document in the master file of the software so that it is readily available should it be required for any exigency. 


\section{Software Registration}

The above section on licensing leads directly to questions regarding registration. In this arena, freeware and open-source software differ from other types of software.

When employing freeware and open source products, one often finds differences between "personal" and "business" use. Many of these packages allow unlimited use of the software as long as its use is strictly personal. In other words, if one downloads a software program, installs it on one's computer, and utilizes it strictly for one's own, non-commercial purposes, then use of the software is free. Personal use refers to all usage that does not generate financial income, such as scrap-booking, creating personal websites, personal blogs, and print jobs such as flyers, posters, and T-shirts for grade schools and local food banks. In this case, one would simply register the software in one's name.

Additionally, charities and other non-profit institutions (such as public, academic, and school libraries) may ordinarily employ freeware and open-source software under the same precincts as individuals, i.e., as personal users.

However, suppose one works at a for-profit enterprise and desires to install the software on his/her office computer. Naturally, since the firm owns all its office computers, one usually registers the software in the company's name. Under such conditions, many freeware packages oblige the user to seek permission from the software owner for such use. Commercial use may also require a fee from the user to the software owner. These caveats also apply to individuals who obtain this software for engagement in their own moneymaking endeavors (such as freelancing). The End-User License Agreement, included with all freeware packages, contains information to guide users in such contingencies.

To avoid worrying about these details, some freeware and open source users simply treat these programs as if they were commercial products and register their use accordingly. This is a safe practice to adopt.

One may be surprised to learn that numerous freeware and open source packages do not require registration at all; others regard user registration as a voluntary exercise. Both stipulations presume non-commercial use of the software.

\section{Locating Freeware and Open-source Software}

Finding freeware and open-source software is a rather simple process. A good place for the neophyte to start searching for them is at Datamation. ${ }^{11}$ Datamation is a periodical that began life in hard copy format in 1957 but morphed into an e-journal in the 1990s (the final print edition appeared in February 1998). To access the list of open source replacements for popular commercial security tools, for example, simply click on the "Open Source" heading of the menu bar on the home page of the website. Scroll down the next page to discover the list of alternatives to 
commercial security software in such categories as Anti-Malware, Backup, and Browsers, until finally, the reader reaches the last category, Web Filtering.

Another important resource comes from PCMag.com. Each month, the site presents a list of the best free software available in a particular category such as firewalls, video conferencing, antivirus, and presentation software. Both freeware and open-source software are included in each category. Last year, experts in the field examined over one hundred free software packages from nine categories. Most of the evaluated packages operate on either Windows 7 or Windows 8, although programs designed for other platforms, such as Mac OS and Android, were included in the lists. Also assayed are free cloud-based Web applications which run in a browser. Note that the lists of free software from 2012 and 2013 are readily available. ${ }^{12}$

John Corpuz's “45 Free and Useful Windows Applications” is a slideshow presenting detailed information on useful applications from a variety of software categories. Embedded within each software description is a link which, when clicked, takes the reader to a window where that software may be downloaded. ${ }^{13}$

dotTech offers one of the most comprehensive lists of freeware for Windows available. This list consists of individual reports organized around nine categories: Cleaning and Maintenance, Communication, Files \& Documents, Work \& Productivity, PDF Tools, Privacy and Security, Multimedia, Network/Internet Tools, and Miscellaneous. Each report is well-written, concise, and features the type of information computer users need. Although I disagree on some of the choices labeled as "best," I cannot but appreciate the exhaustive nature of these documents. Since dotTech continuously revises and expands the number of reports in this set, wise users make periodical visits to this site. ${ }^{14}$

A quick search of the Web will bring one to the Open-source software Directory where one will find open source applications listed by broad categories on the left side of the home page, categories which are then separated into topics before being subdivided by function. To discover network management software, for example, find "Administrators" (category), then "Networking" (topic), and finally, click on "Network Management" (function). In this manner, one will obtain a list of open source products matching this description. ${ }^{15}$

TechRadar provides another register of recommended free software. ${ }^{16}$ This site provides detailed descriptions of seventy-six freeware packages, ranging from productivity software to games. A link takes users to TechRadar's Download Channel where one may read information on freeware and open-source software categorized by function and specific name. ${ }^{17}$

One cannot discuss freeware without mentioning the world's largest supplier of freeware, Microsoft. Veteran users of its Windows operating system and its office suite are undoubtedly cognizant of the templates and other helps the firm offers them. Those implements represent merely a sampling of the valuable and diverse tools the company makes available to the computing world. Microsoft provides access to their abundance of freeware via a download site on the Web; ${ }^{18}$ 
however, many people find the site difficult to navigate. For this reason, many individuals consult a friendlier, third party site that opens the doors to this unique collection of freeware. One of the better examples of these sites can be found at Gizmo's Freeware. ${ }^{19}$

Internet download directories offer an easy and convenient avenue for one to obtain freeware and open-source software. There are a plethora of such sites on the Web; unfortunately, they are not all of equal value. Some sites are simply better than others. Here is a brief list of criteria one may employ to judge these sites:

- Ease of use. Is the site easy to navigate? Does it feature categories that enable one to search for a specific type of software? If it contains categories, does their organization enable one to find software quickly and efficiently?

- Language. Can one easily comprehend the language used to describe the software? Does the language express complex concepts in laypeople's terms or in a manner targeted to information technology professionals?

- Are the software packages accurately described? Do the descriptions detail desirable and undesirable traits of the software? Do they clearly indicate the operating system required by the software? What other prerequisites does the software require for effective operation? Are alternatives to that software specified?

- Are software reviews presented? If so, who wrote the reviews - laypeople or information technology professionals? Do the reviews offer download statistics? Does the site contain a ratings system easily understood by the user?

- Are aids available to help users make optimum use of the software? If aids are available, what format are they in - videos, documentation, other?

- Look for other features which may prove useful, such as: Is there a link to the software's home page? Does the download directory provide access to more than one download site?

Among the download sites meeting these criteria are the following:

CNET Download.com - http://download.cnet.com/windows/

MajorGeeks.com - http://www.majorgeeks.com/

Softpedia - http://www.softpedia.com/

FileHorse.com - http://www.filehorse.com/

Softonic - http://en.softonic.com/

FileHippo.com - http://www.filehippo.com/

FreewareWeb - http://www.freewareweb.com/ 
Freeware Guide - http://www.freeware-guide.com/

Freeware Files - http://freewarefiles.com/

SourceForge - $\underline{\mathrm{http}} \mathrm{/} / /$ sourceforge.net/

This brief list does not even begin to exhaust the number of Internet download directory websites available for use. Frequent visits to these and similar websites will prove amply rewarding.

Another method of finding this software is to simply look for it on the Web via a search engine. To do this most efficiently, one will need the proper name of the software one is trying to obtain. However, if one has that element, one will find this an effective technique of obtaining freeware and open-source software.

How does one seek freeware and open source equivalents to commercial software and shareware? First, one may consult a website entitled AlternativeTo. ${ }^{20}$ This website will present one with a list of alternatives to specifically named commercial software packages. One has only to click on the search dialog box, key in the name of the commercial product one wishes to find alternatives to, and press the search icon. The list of results will include freeware, open-source software, and commercial products. Clicking on the name of the product with transport one to that product's home page, where one will gain additional information on that product and be given an opportunity to download the product onto one's computer.

Secondly, one may consult any of several lists of software equivalents from the Web. One of the better of these registers is "100 Open Source Apps To Replace Everyday Software," by Cynthia Harvey. ${ }^{21}$ This list provides not only names of individual open source projects and the commercial packages they replace but also links to homepages of these projects.

\section{Library-Specific Freeware and Open-source Software}

In the past several years, an increasing number of librarians have turned to freeware and opensource software to help them fulfill the duties they are obligated to discharge.

One of the most renowned examples of library-specific open-source software is Koha, an integrated library system. ${ }^{22}$ Programmers and librarians developed Koha fifteen years ago for the Horowhenua Library Trust in New Zealand. Since then, libraries of all types, including public, academic, and school libraries, have adopted Koha as their integrated library system. Numerous consortia across the globe also employee Koha to meet their needs and those of their users.

One way novices may apprise themselves of this software is to consult websites such as The Creative Librarian, which features a page entitled "Open-Source Software for Libraries," where software is enumerated by library function..$^{23}$ Any type of library, irrespective of the library's size, can utilize the software described on this page. 


\section{Questions Regarding the Use of Freeware and Open-source Software}

All this is not to say that freeware and open-source software do not have their challenges. For instance, in one anecdote I related in the introduction in this paper, I did not name the PDF editor I used at Kansas Wesleyan. That is because the software is no longer available; unfortunately, neither is David Lord's Ascii Chart. Whenever one spots a piece of freeware or open-source software that may be useful, it is imperative to download it immediately. The availability of many packages is limited, and once gone, they are usually difficult, if not impossible, to obtain.

Another concern regards documentation. When I first obtained Picture TimeClock, for example, a complete set of instructions was available from the software provider. That is no longer the case. Although an increasing number of freeware and open source packages offer documentation, many do not. However, as noted earlier in this report, many open-source software products have user groups called communities that exist not only to improve the software but also to provide technical assistance to those who use the software.

Downloading freeware and open-source software presents its own quandaries. Even though most providers of these packages go to great lengths to insure the cleanliness of their product, it is nevertheless true that viruses and malware sometimes attach themselves to this software. Whenever my security software activates during a download, I immediately cease the downloading process and make a note of the site for future reference. Additionally, I always run my security software against all software downloads before installing them in order to keep my system free of any potential threats.

Issues also arise from employing freeware and open-source software in business offices. Individuals bring most of this software into enterprise environments. Since the organization itself doesn't procure this software, the corporation's information technology personnel may be reluctant to provide support. Indeed, the corporation's IT department may not even permit an individual to download any outside software whatsoever onto a system under their domain. Before one attempts to install such software (regardless of type of software) on one's business unit, one should check with the company's IT people to obtain their views on the proposed installation.

\section{Closing Thoughts}

One question remains: why bother with freeware and open-source software? Are librarians searching for new software programs to master? Do they need an additional task to add to their todo lists? Are freeware and open-source software worthy of the attention of already overworked and stressed-out librarians?

Yes, they are worthy of our attention. Why? For three key reasons. First, freeware and open-source software are cost-effective. For no monies whatsoever, freeware and open-source software offer librarians the opportunity to add important new tools to the arsenal of implements at their 
disposal. That means that badly needed funds can be more strategically used by the library to help enable it to fulfill its mission to its clientele.

Secondly, freeware and open-source software enable librarians to make increased use of computer hardware. Computers are machines: they require software to not only tell them which tasks to execute but also to provide instructions for performing those tasks. With this software, the range of computer capabilities not only expands in terms of numbers but also increases in terms of efficiency. The bottom line: freeware and open-source software enhance the value of computer hardware to the library and its patrons.

Finally, with the assistance of freeware and open-source software, librarians become better librarians. They manage their time more effectually, make better use of the resources at their disposal, and elevate the degree of customer service at all levels of the organization. Freeware and open-source software can expedite the handling of routine assignments and make possible the fulfillment of other jobs that, due to time and human limitations, are difficult, if not impossible, to address. Freeware and open-source software are good for librarians, good for the library, and good for those who depend on the library for the fulfillment of their information needs. They truly foster what many individuals refer to as a "win-win situation" in the world of information acquisition, organization, preservation, and retrieval.

\section{URLS CITED}

1. "Picture TimeClock," Guia International Corporation, http://workschedules.com/Store/Product/Picture Time Clock.aspx.

2. “The Open Source Definition," Open Source Initiative, http://opensource.org/docs/osd.

3. "Public-Domain Software," Webopedia: Online Tech Dictionary for IT Professionals, http://www.webopedia.com/TERM/P/public domain software.html.

4. "Fast and Fluid for Windows 7: Get Internet Explorer 11," Microsoft, http://windows.microsoft.com/en-US/internet-explorer/download-ie.

5. "Firefox Web browser," Mozilla, http://www.mozilla.org/en-US/firefox/new/.

6. "CCleaner," Piriform, http://www.piriform.com/ccleaner.

7. "Audacity," Audacity: Free Audio Editor and Recorder, http://audacity.sourceforge.net/.

8. "Apache," The Apache HTTP Server Project, http://httpd.apache.org/.

9. "A Quick Guide to GPLv3," GNU Operating System, http://www.gnu.org/licenses/quick-guidegplv3.html.

10. “GNU General Public License," GNU Operating System, https://www.gnu.org/licenses/gpl3.0.txt. 
11. "About Us: Datamation," Datamation, http://www.datamation.com/about/.

12. "The Best Free Software," PCMag.com, http://www.pcmag.com/article2/0,2817,2381528,00.asp.

13. "45 Free and Useful Applications," Tom's Guide: Tech for Real Life, http://www.tomsguide.com/us/pictures-story/286-39-best-free-windows-apps.html.

14. "Best Windows Free Software," dotTech, http://dottech.org/best-free-windows-software.

15. “Open-source software Directory," http://www.opensourcesoftwaredirectory.com/.

16. "The Best Free Software for your PC: Essential PC Programs You Should Download Today," TechRadar, http://www.techradar.com/us/news/software/the-best-free-software-for-yourpc-1221029.

17. “Newest Downloads," TechRadar, http://www.techradar.com/us/downloads.

18. "Microsoft Download Center," Microsoft Corporation, http://www.microsoft.com/enus/download/.

19. “Best Free Microsoft Downloads," Gizmo's Freeware: the Best Freeware Reviewed and Rated, http://www.techsupportalert.com/content/best-free-microsoft-downloads.htm.

20. "AlternativeTo," http://alternativeto.net/.

21. "100 Open Source Apps To Replace Everyday Software," Datamation, http://www.datamation.com/open-source/100-open-source-apps-to-replace-everydaysoftware-1.html.

22. "Koha Library Software," Official Website of Koha Library Software, http://kohacommunity.org/.

23. "Open-Source Software for Libraries," The Creative Librarian, http://creativelibrarian.com/library-oss/. 